\title{
Simulation of Electric Faults in Doubly-Fed Induction Generators Employing Advanced Mathematical Modelling
}

\author{
Sebastian Martens, Nenad Mijatovic, Joachim \\ Holbøll \\ Centre of Electric Power and Energy \\ Department of Electrical Engineering \\ Technical University of Denmark \\ 2800 Lyngby, Denmark
}

\author{
Georgios A. Skrimpas, Christian W. Sweeney \\ Remote Monitoring Group \\ Brüel \& Kjær Vibro A/S \\ 2850 Ncrum, Denmark
}

\begin{abstract}
Efficient fault detection in generators often require prior knowledge of fault behavior, which can be obtained from theoretical analysis, often carried out by using discrete models of a given generator. Mathematical models are commonly represented in the DQ0 reference frame, which is convenient in many areas of electrical machine analysis. However, for fault investigations, the phase-coordinate representation has been found more suitable. This paper presents a mathematical model in phase coordinates of the DFIG with two parallel windings per rotor phase. The model has been implemented in Matlab and its properties in context of fault simulations and investigations has been investigated. Some of the most common faults have been simulated, namely broken rotor bars or windings, dynamic eccentricities and stator phase winding short circuits. These fault conditions propagate to the stator current as undesired spectral components, which can be detected by applying frequency spectrum analysis.

Index Terms - Wind turbine generators, doubly-fed induction generators, mathematical modelling, fault investigations
\end{abstract}

\section{Introduction}

Wind power has proven an efficient sustainable energy source and the global market has grown considerably within the last decade or so. Large off-shore wind farms consisting of several multi megawatt wind turbines have been introduced and established, and today, wind turbines contribute with a considerable energy production [1], [2]. A common choice of wind turbine generator has for many years been the Doubly-Fed Induction Generator (DFIG). This is due to its properties in the context of variable-speed wind turbine systems and the geared DFIG configurations account for approximately $50 \%$ of the offshore wind turbines, and more than $65 \%$ of the total wind power market share [3], [4], [5].

The fast growth of wind energy technology has introduced certain demands on the reliability of the wind turbines. Today, the downtime is kept low thanks to frequent and fast maintenance rather than the reliability itself and this fact have an influence on the total expenses associated with the wind turbine. The estimated ex- penses of a wind turbine with a lifetime of 20-years, including operation and maintenance, are $10-15 \%$ and $20-25 \%$ of the total income for onshore and offshore wind turbines, respectively. [5], [6]. Hence, conditionbased maintenance strategies have become an important asset of wind turbine manufacturers and operators in order to improve the operation and energy production, thereby lowering the expenses.

It is important that faults, defects and other problems affecting the normal operation of these wind turbines are detected and solved before they propagate, and cause serious damage to the turbine. In order to detect a fault and determine its causes, the diagnostic system has to rely on known fault characteristics, typically obtained from theoretical analysis and tests, and measured machine data. The usability of various measurable machine quantities has been explained in [7] and [8].

Theoretical analysis of electric machines is often carried out on the basis of discrete models. The Finite Element Method (FEM) has been utilized in various researches on fault diagnostics with satisfying results, e.g. [9], [10]. FEM provides the opportunity of utilizing detailed knowledge of the machine design and structure in order to obtain a highly detailed and unique model. Some drawbacks of the FEM are the computational time, lack of flexibility and potential modeling difficulties. Mathematical models are widely used in the context of control systems and in the investigation of grid faults. The models are based on a higher level of abstraction compared to FEM models and non-linearities are usually neglected. Compared to FEM models, mathematical models tend to be more flexible and computationally lighter, but their usability in the context of fault condition analysis is more limited. However, mathematical models have been used for extensive analysis of machine behaviour with satisfying results [11].

This paper presents an analysis of the properties of mathematical models in the context of fault analysis. This has been done on the basis of simulations of different electric and mechanical fault conditions in wind turbine DFIG's. Some faults are more often encountered than others, and some faults are more severe than others. Sections 2 and 3 explains the basic design and functionality of a common wind turbine DFIG's and presents a mathematical model, which has been used for fault investigations. Different fault conditions are pre- 
sented together with simulation results in sections 4 and 5 .

\section{The General Design, Construction and Functional- ity of a DFIG used in Wind Turbines}

The DFIG is fed with AC currents both at the stator and the rotor, hence the name "Doubly-Fed". The stator contains three-phase, sinusoidally distributed windings, embedded in slots around the inner periphery of the stator. The stator core is made of thin silicon steel laminations, stacked on top of one another and insulated in order to minimize iron losses caused by eddy-currents. The rotor of the DFIG is equipped with three-phase windings similar in distribution to the stator windings. A common rotor design of a DFIG used in wind turbines is given by Fig. 1, where each rotor phase has been equipped with two parallel windings per phase, giving a total of six rotor windings. The windings are embedded into slots on the exterior periphery of the rotor core and connected separately to external wires through slip-rings on the rotor-shaft. An outline of the magnetomotive force (MMF) created by the rotor windings in this configuration has been provided in [5]. Both stator and rotor windings are held in place by wedges. With these winding configurations, the air-gap can be considered uniform and thereby there is no saliency in the generator. The stator is connected directly to the grid and the rotor is connected to a three phase electric source. By adjusting the amplitude and frequency of the currents fed to the rotor, it is possible to keep the voltage and frequency of the stator constant [3], [4], [12]. The DFIG has three modes of operation depending on the rotational speed of the rotor shaft:

- Sub-synchronous speed

- Synchronous speed

- $\quad$ Super-synchronous speed

The synchronous speed is given as follows:

$$
\square_{\square}=\frac{60 \square \square}{\square}[\square \square \square]
$$

Here, $\square_{\square}$ denotes the stator, or grid frequency and $\square$ is the number of pole-pairs. The slip is defined as the difference between the synchronous speed $\square_{\square}$ and the electromechanical speed $\square_{\square \square}$ of the rotor:

$$
\square=\frac{\square_{\square}-\square_{\square \square}}{\square_{\square}}
$$

In wind turbine systems, the rotor voltage of the DFIG is controlled by a medium scale power converter configuration, where two converters are placed back-toback and separated by a DC-link. The rated power of the converters is only $30 \%$ of the nominal power and for variable speed systems where the requirements to the speed range of the generator are small, the speed variations are typically within $+/-30 \%$ of the synchronous speed [1], [13]. When the DFIG runs at sub- synchronous speed, the stator continues to deliver power to the grid and the rotor absorbs power from the grid. When the DFIG runs at super-synchronous speed both the rotor and the stator deliver power to the grid. Supersynchronous speed operation is usually considered as a brief overload condition caused by wind gusts and if the conditions persists, the pitch-control dump the blade load. The minimum sub-synchronous operation is determined by the available wind power at low speed and the economic aspect of operation under these circumstances.

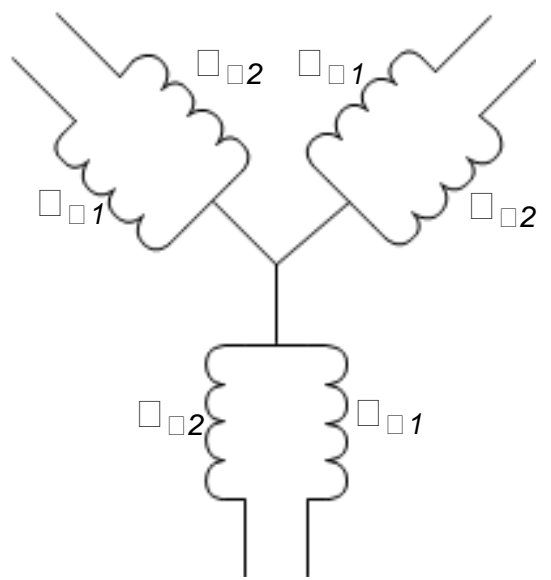

Fig. 1 A Common rotor winding configuration of a DFIG used in wind turbines.

\section{Mathematical Model of the Wind Turbine DFIG}

A general mathematical model of a doubly-fed induction machine is well established in literature [11], [14] and [15]. The model is based on voltage equations in terms of machine variables, also known as the phasecoordinate model and is in many circumstances considered to be too cumbersome to work with [12]. The $\square \square 0$ obtained from Park's transformation of the phasecoordinate model, is thus the most common and preferred mathematical model. For fault simulation purposes, the phase-coordinate model is in many ways more advantageous compared to the $\square \square 0$ model, because of the possibilities of changing machine parameters according to an imposed fault and simulation of unbalanced conditions.

Since the common mathematical model of a DFIG in phase coordinates does not consider the specific case of having two windings per rotor phase, it has been necessary to expand the common model with an extra equation taking the secondary rotor phase windings into account. This also introduces additional flux-linkages. The equations of the expanded model are given by the following expressions:

$$
\begin{gathered}
\square_{\square}=\square_{\square} \square_{\square}+\frac{\square \square_{\square}}{\square \square} \\
\square_{\square 1}=\square_{\square 1} \square_{\square 1}+\frac{\square \square_{\square 1}}{\square \square} \\
\square_{\square 2}=\square_{\square 2} \square_{\square 2}+\frac{\square \square \square 2}{\square \square}
\end{gathered}
$$

where the voltages and currents are vectors according to: 


$$
\begin{gathered}
\left(\square_{\square}\right)^{\square}=\left[\begin{array}{lll}
\square_{\square \square} & \square_{\square \square} & \square_{\square \square}
\end{array}\right] \\
\left(\square_{\square 1}\right)^{\square}=\left[\begin{array}{lll}
\square_{\square \square 1} & \square_{\square \square 1} & \square_{\square \square 1}
\end{array}\right] \\
\left(\square_{\square 2}\right)^{\square}=\left[\begin{array}{lll}
\square_{\square \square 2} & \square_{\square \square 2} & \square_{\square \square 2}
\end{array}\right]
\end{gathered}
$$

$f$ represents the machine quantities i.e. voltages and currents, respectively and the subscripts denotes the machine component and phase. The resistances $\square_{\square}$, where $\square=\square, \square 1, \square 2$ are diagonal matrices, containing the winding resistances. The flux-linkages can be described by means of inductances and in matrix format, they can be presented as:

$$
\left[\begin{array}{c}
\square_{\square} \\
\square_{\square 1} \\
\square_{\square 2}
\end{array}\right]=\left[\begin{array}{ccc}
\square_{\square \square} & \square_{\square \square 1} & \square_{\square \square 2} \\
\square_{\square 1 \square} & \square_{\square 1 \square 1} & \square_{\square 1 \square 2} \\
\square_{\square 2} & \square_{\square 2 \square 1} & \square_{\square 2 \square 2}
\end{array}\right] \cdot\left[\begin{array}{c}
\square_{\square} \\
\square_{\square 1} \\
\square_{\square 2}
\end{array}\right]
$$

Each element of the three-by-three matrix in equation (10) (henceforth noted as the induction matrix) is also a three-by-three matrix containing the inductances of each machine winding. The first row, columns two-three and the first column, row two-three of the induction matrix contains the time varying mutual inductances of the stator and rotor, whereas the rest represents stationary self and mutual inductances, as well as leakage inductances. It is common to neglect the mutual leakage inductances between windings on the same machine member. Combining equations (3) to (10) yields the following matrix representation. The vectors containing the voltages $\square$ and currents $\square$, respectively, are column vectors, the resistances $\square$ is a nine-by-nine diagonal matrix and the inductance matrix $\square$ is also nine-by-nine:

$$
\begin{aligned}
& {\left[\begin{array}{c}
\square_{\square} \\
\square_{\square 1} \\
\square_{\square 2}
\end{array}\right]=\left[\begin{array}{c}
\square_{\square} \\
\square_{\square 1} \\
\square_{\square 2}
\end{array}\right] \cdot\left[\begin{array}{c}
\square_{\square} \\
\square_{\square 1} \\
\square_{\square 2}
\end{array}\right]}
\end{aligned}
$$

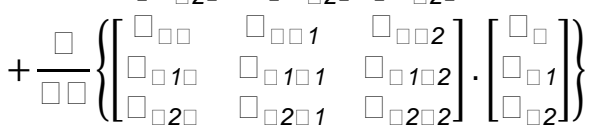

Choosing the machine currents as state-variables and rearranging, yields the following state-space representation of the DFIG phase-coordinate model:

$$
\begin{aligned}
& \frac{\square \square}{\square \square}=-\square^{-\square}\left[\square+\frac{\square \square_{\square \square}}{\square \square} \cdot \frac{\square \square\left(\square_{\square \square}\right)}{\square \square_{\square \square}}\right] \square \\
& +\square^{-\square} \square
\end{aligned}
$$

Here, $\square_{\square \square}$ is the electromechanical angular position. Equation (12) is constrained by $\square$ being non-singular, since the inverse of $\square$ is needed in order to obtain the time derivatives of the machine currents.

It is common to make an assumption of healthy working conditions, making it possible to consider several of the inductances as equal and it is also common to refer the rotor quantities to the stator. For fault simulation purposes, all elements of the vectors and matrices should be available for alterations. As a last comment, it is important to note that the model has been derived on the basis of an idealized magnetic system and that several aspects of the DFIG design have not been taken into account. However, the model is adequate for many purposes, including simulations of a wide range of fault conditions. Parameters can be modified and changed in specific patterns to incorporate non-linearities and other machine effects, as has been done in [11].

\section{Common Fault conditions in Wind Turbine DFIG's}

The major fault types of electric machines can in a wide sense be classified as listed in Table 1, [16].

Table 1 - Major fault types in electric machinery

- $\quad$ Stator faults resulting in short and open circuits of one or several stator windings

- Abnormal connections of the stator windings

- Broken rotor bars or cracked end rings (as for cage rotor induction machines)

- $\quad$ Static and/or dynamic air-gap irregularities (e.g. as a result of rotor eccentricities)

- Bent rotor shaft much alike a dynamic eccentricity, resulting in contact or rubbing of the stator and rotor causing severe damage to windings, wedges etc. of the stator and rotor

- Rotor faults resulting in short and open circuits of one or several rotor field windings

- Bearing related faults

- $\quad$ Stator and rotor magnetic wedge faults (e.g. flying wedges)

- Damaged or bad connected rotor cables

- $\quad$ Slip-ring issues

- De-magnetization of permanent magnets

- Loose, missing or broken permanent magnets

In [6] and [9] some statistics on the most frequent fault conditions encountered in induction machines have been presented. For wind turbine generators, bearing related faults are the most common with $40 \%$, followed by stator related faults $38 \%$ and rotor related faults $10 \%$. It has been shown that the majority of these fault types are traceable to the stator current, where the faults show up as unwanted frequency components. However, some faults may be difficult to distinguish as the faults results in similar conditions and in addition, the faults are typically time variant, [6], [8]. Hence, to ensure fault detectability, a combination of different diagnostic methods is needed. Using the stator current as the measured machine quantity is practical in the context of model simulations, since the machine currents usually are directly available as state variables or obtainable through mathematical computations.

The fault simulations presented in the last part of this paper has been based on how frequent a specific fault condition occurs, which is given above as a percentage. Thus, a stator short circuit fault, a broken or disconnected rotor winding and a dynamic eccentricity caused by a faulty bearing has been considered.

\section{Simulations of Common DFIG Fault Conditions}


The mathematical model has been implemented in Matlab. At each time step, the induction matrix $\square$ and its derivative, the voltages and resistances (which is basically constant) are calculated. Then, equation (12) is calculated and the time derivatives of the machine currents are integrated by means of the Dormand-Prince "ode45", which is a medium order solver for non-stiff problems and with a medium order of accuracy. Only the steady-state behavior has been considered.

The model presents a 2MW, 4-pole wind turbine DFIG, operated at rated power and at a constant speed of 1395 revolutions per minute (RPM), giving a constant 0.07 slip. The stator voltage is $690 \mathrm{~V}_{\mathrm{RMS}}$ at $50 \mathrm{~Hz}$. The rotor voltage supplied by the power converter has been calculated accordingly, giving a stator current of approximately $1650 \mathrm{~A}_{\mathrm{RMS}}$. Both stator and rotor has been assumed to be connected in star. Initial conditions have been assumed to be zero and the simulation time-step is $10 \mu \mathrm{s}$. All frequency spectrums have been obtained with added white Gaussian noise and a signal to noise ratio (SNR) of 0.01 at steady-state.

\subsection{DFIG Running Under Healthy Conditions}

A simulation of the DFIG operating under healthy conditions has been carried out in order to verify the behavior and obtain a reference for faulty conditions. The results are given by Figs. 2 to 4 .

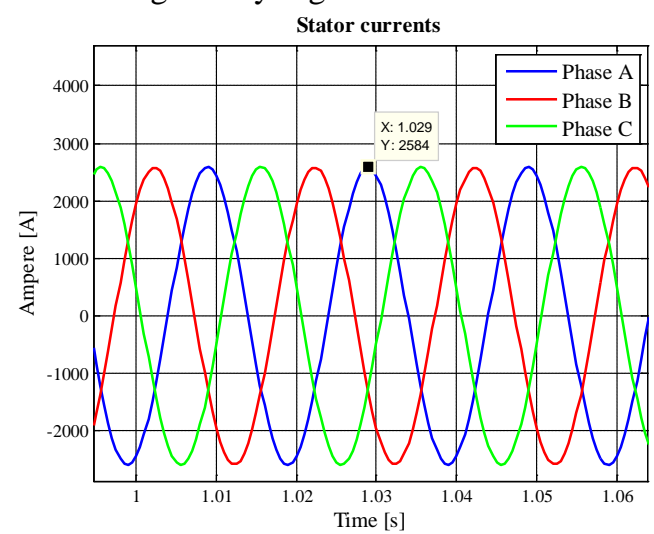

Fig. 2 Stator currents in steady-state under normal machine operation. The slip is 0.07 . The current waveforms behave as expected.



Fig. 3 Rotor currents in steady-state under normal machine operation. The slip is 0.07 . A small phase shift between the currents of each phase winding would be expected, since they are displaced by a small spatial angle, affecting their mutual inductance. However, the difference is small.

The frequency spectrum provided by Fig. 4 shows a fundamental component of $50 \mathrm{~Hz}$ as expected. The system has been assumed linear and thus harmonics due to non-linearities are not present. Non-linear behavior can be implemented if desired, but has been saved for future work. As an example, saturation can be emulated by making $\square_{\square}$ dependent on the current.



Fig. 4 Frequency spectrum of the stator current in steady-state under normal machine operation. The slip is 0.07 .

\subsection{Broken or Disconnected Rotor Winding}

This fault condition could be due to a broken brush or cable (external faults), but a broken rotor winding could also be caused by other internal faults. The fault condition can be detected by stator current spectrum analysis, but the condition can also be detected by other means, for instance vibrational analysis as proven in [5]. A broken rotor winding has been simulated by effectively reducing the system order, thus avoiding a singularity condition in the inductance matrix $\square$.

Figs. 5 and 6 show the frequency spectrums of stator and rotor currents respectively. As the slip changes, the fault component appears at different frequencies, suggesting a direct dependency on the slip.

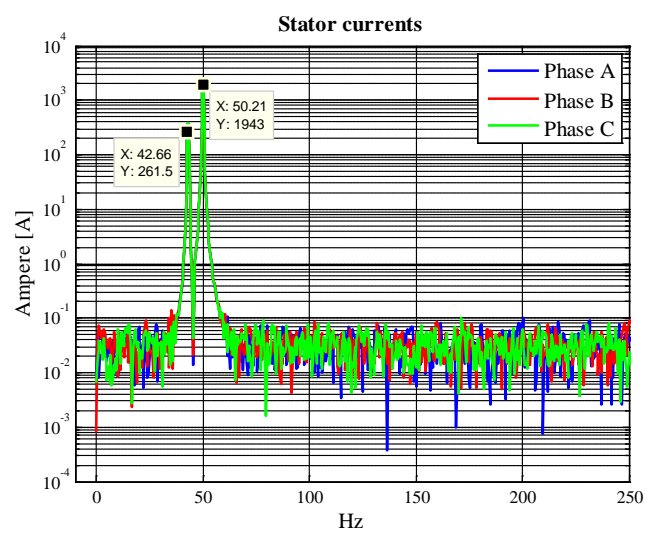

Fig. 5 Frequency spectrum of the stator current in steady-state. One rotor winding has been disconnected. The slip is 0.07. A fault component shows a spectral component matching the 
frequency of the fundamental frequency minus two times the slip-frequency.



Fig. 6 Frequency spectrum of the stator current in steady-state. One rotor winding has been disconnected. The slip is -0.07 . A fault component shows a spectral component matching the fundamental frequency plus two times the slip-frequency.

\subsection{Short-Circuit in a Stator Winding}

A short-circuit between several turns of one stator winding is typically caused by insulation breakdown due to several possible causes, including temperature rises caused by other faults. This fault condition would change the impedance of the winding, thereby affecting the phase current. The resulting wire can be considered to be shortened, affecting the resistance and the winding inductance would change as well, since the number of turns effectively changes. The fault has been imposed by altering the specific inductances and the resistance of the phase A winding.

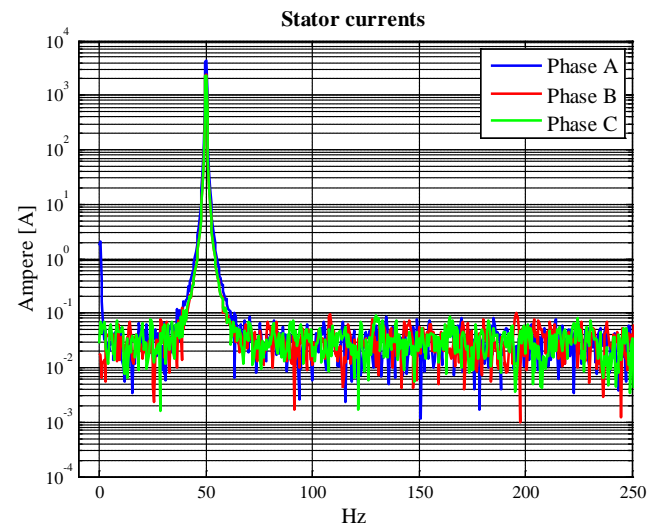

Fig.7 Frequency spectrum of the stator current in steady-state under normal machine operation. The slip is 0.07. A low frequency component is visible, which may be a DC value.

Changes in the impedance cause an unbalanced load condition, which can be seen from the frequency spectrum of Fig. 7. In addition, a DC component has been induced in the unbalanced phase current. Operating the machine at negative slip does not seem to have an influence on the result, but this has been assessed to be due to limitations in the model.

\subsection{Dynamic Eccentricity of the Rotor}

Dynamic eccentricities results in time dependent variations of the air-gap. These variations can be caused by faults in the bearings, misalignment of the rotor shaft or similar conditions. If the case is a slightly bend rotor shaft, the air-gap will decrease in the direction of the bend and at the same time increase in the opposite direction of the bend. This change is cyclic with the mechanical speed of the rotor shaft and affects all phases. Figs. 8 and 9 presents the stator frequency spectrum at a positive and negative slip, respectively, obtained from simulations with a cyclically small variation in air-gap length. Considering the low frequency component, it is seen that a downwards shift in frequency occurs when the slip changes from positive to negative. This change corresponds to the slip-frequency. The magnitude increases slightly. The high frequency component moves up in frequency with an amount corresponding to the slip-frequency and the magnitude increases with a considerable amount, compared to the low frequency component. Considering the fundamental frequency component, it does not change in frequency as expected. However, a decrease in magnitude is seen at negative slip, compared to positive slip.

It has been assessed that model limitations and some uncertainty regarding the fault imposing method have an impact on the results. As such, the changes in frequency component magnitudes depending on the slip are somewhat uncertain. Thus, further investigations concerning eccentricity fault behavior are needed.

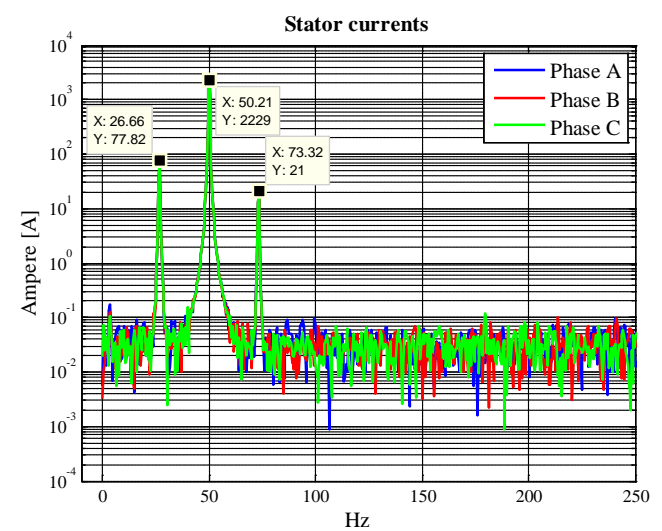

Fig. 8 Frequency spectrum of the stator current in steady-state under normal machine operation. The slip is 0.07 . 




Fig. 9 Frequency spectrum of the stator current in steady-state under normal machine operation. The slip is -0.07 .

\section{Conclusion}

In this study, a mathematical model of a wind turbine DFIG with two windings per rotor phase has been presented and its properties within fault simulations and investigations have been assessed. In addition, fault types arising from electrically and mechanically related defects have been simulated in order to investigate fault behavior. It has been shown that the mathematical model shows satisfying possibilities within fault investigations and it has been shown that faults tend to be dependent on the slip frequency, which makes it necessary for the diagnostic system to take the operating speed into account.

Further work includes extensive fault investigations, addition of specific non-linearities to the model and investigation of the model functionality with other generator specifications, operating modes and data.

\section{References}

[1] S. Müller, M. Deicke \& Rik W. De Doncker "Doubly Fed Induction Generator Systems for Wind Turbines” IEEE Industry Apllications Magazine, May - June 2002, www.IEEE.org/IAS.

[2] Z.Q. Zhu and Jiabing $\mathrm{Hu}$ "Electrical machines and power-electronic systems for high-power wind energy generation applications Part I - market penetration, current technology and advanced machine systems", Compel- The International Journal for Computation and Mathematics in Electrical and Electronic Engineering - 2013, Volume 32, Issue 1, pp. 7-33.

[3] Gonzalo Abad, Jesús López, Miguel A. Rodríguez, Luis Marroyo \& Grzegorz Iwanski, Doubly Fed Induction Machine - Modeling and Control for Wind Energy Generation, IEEE Press, John Wiley \& Sons Inc, 111 River Street, Hoboken, NJ 07030, 1st Edition, 2011, 625 pages.

[4] Bin Wu, Yongqiang Lang, Navid Zargari \& Samir Kouro, Power Conversion and Control of Wind En- ergy Systems, IEEE Press, John Wiley \& Sons Inc, 111 River Street, Hoboken, NJ 07030, 1st Edition, 2011, 453 pages.

[5] Georgios A. Skrimpas, Christian W. Sweeney, Bogi B. Jensen, Nenad Mijatovic, Joachim Holbøll, "Analysis of generator bearing vibration data for diagnosing rotor circuit malfunction in DFIGs", 2014 International Conference on Electrical Machines (ICEM) - 2014 International Conference on Electrical Machines (ICEM) — 2014, pp. 17461751, pp. 1746-1751

[6] Lu Bin, Yaoyu Li, Xin Wu and Zhongzhou Yang "A Review of Recent Advances in Wind Turbine Condition Monitoring and Fault Diagnosis”, 2009 IEEE Power Electronics and Machines in Wind Applications - 2009, pp. 109-115

[7] Anouar Belahcen, Javier Martinez, Toomas Vaimann, "Comprehensive Computations of the Response of Faulty Cage Induction Machines", 2014 International Conference on Electrical Machines (ICEM) - 2014 International Conference on Electrical Machines (ICEM) - 2014, pp. 15101515, pp. 1510-1515

[8] Bram Corne, Colin Debruyne, Patrick De Baets and Jan Desmet, "Stator Current Measurements as a Condition Monitoring Technology - The-State-ofthe-Art”, 2014 International Conference on Electrical Machines (ICEM) — 2014 International Conference on Electrical Machines (ICEM) - 2014, pp. 1659-1665, pp. 1659-1665

[9] S.K. Sahoo, P. Rodriguez, M. Sulowicz, "Comparative Investigation of Fault Indicators for Synchronous Machine Failures”, 2014 International Conference on Electrical Machines (ICEM) - 2014 International Conference on Electrical Machines (ICEM) - 2014, pp. 1503-1509, pp. 1503-1509

[10] V. Fireteanu, P. Lombard, A.I. Constantin, “Detection of a Short-Circuit Fault in the Stator Winding of Induction Motors through Neighboring Magnetic Field Harmonics”, 2014 International Conference on Electrical Machines (ICEM) — 2014 International Conference on Electrical Machines (ICEM) - 2014, pp. 1555-1561, pp. 1555-1561.

[11] L.A. Fajardo R., F. Iov, F. Blaabjerg and A.D. Hansen, "Advanced Induction Machine model in Phase Coordinates for Wind Turbine Application”, Proceedings of IEEE International Electric Machines and Drives Conference, IEMDC 2007 2007, Volume 2, pp. 1189-1194

[12] Ion Boldea, Variable Speed Generators, CRC Press, Taylor \& Francis Group, 6000 Broken Sound Parkway NW, Suite 300, Boca Raton, FL 334872742, 1st Edition, 2006, (516) pages

[13] John Fletcher and Jin Yang (2010) "Introduction to the Doubly-Fed Induction Generator for Wind Power Applications, Paths to Sustainable Energy”, Dr Artie Ng (Ed.), ISBN: 978-953-307-401-6, InTech, DOI: 10.5772/12889.

[14] Paul Krause, Oleg Wasynczuk, Scott Sudhoff \& Steven Pekarek, Analysis of Electric Machinery and 
Drive Systems, IEEE Press, John Wiley \& Sons Inc, 111 River Street, Hoboken, NJ 07030, $3^{\text {rd }}$ Edition, 2013, 659 pages.

[15] P. Pillay, V. Levin, "Mathematical models for induction machines" Conference Record, Industry Applications Society, IEEE-IAS Annual Meeting — 1995, Volume 1, pp. 606-616 vol.1.

[16] Subhasis Nandi, Member, IEEE, Hamid A. Toliyat, Senior Member, IEEE, and Xiaodong Li, Student Member, IEEE, "Condition Monitoring and Fault Diagnosis of Electrical Motors-A Review”, IEEE Transactions on Energy Conversion - 2005, Volume 20, Issue 4, pp. 719-729 